\title{
Towards Prognostics for Electronics Components
}

\author{
Bhaskar Saha \\ MCT, Inc. \\ NASA Ames Research Center \\ MS 269-3 \\ Moffett Field, CA 94035 \\ 650-604-4379 \\ bhaskar.saha@nasa.gov
}

\author{
Jose R. Celaya \\ RIACS \\ NASA Ames Research Center \\ MS 269-4 \\ Moffett Field, CA 94035 \\ 650-604-4596 \\ Jose.R.Celaya@mail.arc.nasa.gov \\ Philip F. Wysocki \\ ASRC Aerospace Corp. \\ NASA Ames Research Center \\ MS 213-15 \\ Moffett Field, CA 94035 \\ 650-604-0122 \\ philip.wysocki@nasa.gov
}

\author{
Kai F. Goebel \\ NASA Ames Research Center \\ MS 269-4 \\ Moffett Field, CA 94035 \\ 650-604-4204 \\ kai.goebel@nasa.gov
}

\begin{abstract}
Electronics components have an increasingly critical role in avionics systems and in the development of future aircraft systems. Prognostics of such components is becoming a very important research field as a result of the need to provide aircraft systems with system level health management information. This paper focuses on a prognostics application for electronics components within avionics systems, and in particular its application to an Isolated Gate Bipolar Transistor (IGBT). This application utilizes the remaining useful life prediction, accomplished by employing the particle filter framework, leveraging data from accelerated aging tests on IGBTs. These tests induced thermal-electrical overstresses by applying thermal cycling to the IGBT devices. In-situ state monitoring, including measurements of steady-state voltages and currents, electrical transients, and thermal transients are recorded and used as potential precursors of failure. ${ }^{1,2}$
\end{abstract}

\section{TABLE OF CONTENTS}

1. INTRODUCTION ...1

2. BACKGROUND. ....1

3. PREDICTION ALGORITHMS ...................................................2

4. EXPERIMENT DETAILS ..........................................................3

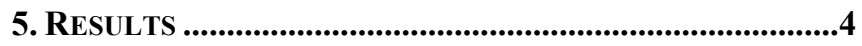

6. CONCLUSIONS ……………………….........................................

ACKNOWLEDGEMENTS ...............................................................5

REFERENCES …............................................................................5

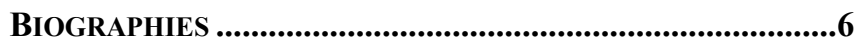

\section{INTRODUCTION}

Predicting the Remaining Useful Life (RUL) of electronic components like IGBTs, MOSFETs (metal-oxidesemiconductor field-effect transistor), and BJTs (bipolar junction transistor) is one of the most challenging frontiers of Prognostic and Health Management systems. These

${ }^{1}$ U.S. Government work not protected by U.S. copyright.

${ }^{2}$ IEEEAC paper \# 1337, Version 2, Updated January 9, 2009. components form the backbone of avionics systems that play an ever-increasing critical role in on-board, autonomous functions for vehicle controls, communications, navigation, and radar systems.

During high-voltage operation, large internal electrical fields build up within power devices, which can increase the rate of degradation. For prognostic purposes the externally observable metrics of the device, such as collector current/voltage measurements during duty cycle on/off states of the IGBT that show degradation trends towards thermal runaway or latch-up, are correlated to physics-offailure mechanisms like hot carrier injection, electromigration, etc., and used in a particle filtering (PF) framework to carry out RUL prediction.

\section{BACKGROUND}

A study of failure precursors for prognostics of IGBTs has been presented in [1]. This work focused on devices that had been aged under thermal overstress [3] resulting in identified precursors of failure that could be measured and computed from external signals. An accelerated aging, characterization and operational scenario simulation system was presented in [2]. This system allowed for aging under different operational conditions providing thermal and electrical overstress to devices, while monitoring in-situ key operational parameters that could serve as precursors of failure. The development of physics based models of aging and fault progression has also been considered. The work presented in [3] focuses on modeling the aging effects at a system level, by studying electrical power drivers using IGBTs.

\section{Failure Mechanisms Review}

Some major intrinsic faults relevant to transistor physics include dielectric breakdown, hot carrier injection, and electromigration $[4,5]$. Some major extrinsic faults relevant 
to transistor packaging-, include contact migration, wire lift, die solder degradation and package delamination $[4,6]$.

Dielectric breakdown occurs when a strong electric field induces a current channel through a previously insulated medium. Acute dielectric breakdown is typically the result of electrostatic discharge (ESD) and junction over-voltage. Time Dependent Dielectric Breakdown (TDDB) refers to the breakdown of gate oxide caused by chronic defect accumulation in the $\mathrm{SiO}_{2}$ insulator. TDDB is shown to be advanced by increases in electric field strength [5]. A strong electric field may induce $t$ energy into an electron or a hole which then becomes a "hot carrier" due to the high kinetic energy stored [4]. Hot carriers have sufficient energy to tunnel and become trapped in gate oxide, and they are the primary cause of TDDB and contribute to device failures under normal operating conditions. Electromigration is a result of high current densities in silicon interconnects causing migration of metals. Formation of metal voids on interconnects can cause open circuits or high resistive paths, which in turn can result in poor performance or circuit malfunction [5].

Contact migration forms metal voids between external contact metals and the silicon. As metal voids grow, the aluminum or other metals can diffuse down to the silicon. This in turn can cause metal spikes to form deep in the silicon region which results in shorting the $p-n$ junctions [7]. Wire lift occurs when the bond between the package wires connecting to the silicon die fail. Wire lift has been identified as a dominant failure mode in high power IGBTs [6]. Die solder degradation is another prominent package related fault. Solder attaching the silicon die and package heatsink develop cracks and voids due to thermal expansion mismatch between materials during expansion and contraction $[6,8]$.

\section{Accelerated Aging Methodologies}

Thermal stress and electrical stress are the most common aging methodologies. Thermal cycling and chronic temperature overstress are prevalent thermal stress methods, with thermal cycling among the most prevalent accelerated aging methodology in electronics. Thermal cycling subjects devices to rapid changes in temperature differentials causing thermal expansion and contraction. Die solder degradation and wire lift are associated strongly with this aging method. Thermal overstress, another prevalent method, subjects devices to high temperatures for extended periods of time. TDDB is accelerated under high temperatures [9] and transistors have exhibited temperature dependant lifetimes accelerated by this mechanism [10]. IGBTs aged with self heating have shown changes in current ringing characteristics during switching [3].

Electrical overstress can be induced though transient and steady-state methods. Transient methods include electro static discharge (ESD), inductive switching and electromagnetic pulses. ESD is a leading cause of gate oxide failure [11] and hard switching of inductive loads, causeing voltage spikes which can cause significant damage to drain-source junctions [12]. Steady-state methods include chronic over-voltage and over-current. Applying high gate voltages [13], setting gate voltage $\left(V_{g}\right)$ to maximize drain current [14], and applying current overstress across the drain [15] have been shown to induce hot carrier and TBBD [9].

\section{Prediction Algorithms}

Particle filtering is a sequential Monte Carlo (SMC) technique for implementing a recursive Bayesian filter using Monte Carlo simulations. It is primarily used for state estimation and tracking. The mathematical formulation for PF methods have been discussed in [16]. The basic idea is to develop a nonparametric representation of the system state probability density function (pdf) in the form of a set of particles with associated importance weights. The particles are sampled values from the unknown state space and the weights are the corresponding discrete probability masses. As the filter iterates the particles are propagated according to the system state transition model, while their weights are updated based upon the likelihood of the measurement given the particle values. Resampling of the particle distribution is done when needed in order to prevent the degeneracy of the weights. For state prediction purposes the same PF framework can be used by running only the model-based particle propagation step until the predicted state value crosses some predetermined end-of-life threshold. The predicted trajectory of each particle then generates an estimate of RUL, which can be combined with the associated weights to give the RUL pdf. The process is broken down into an offline learning part, and an online tracking and prediction part. During offline analysis, regression is performed to find representative ageing curves. Exponential growth models, as shown in Eqn. 1, are then fitted on these curves to identify the relevant decay parameters like $C$ and $\lambda$ :

$$
\theta=C \exp (-\lambda t)
$$

where $\theta$ is an internal model parameter of interest. More details of the PF framework used here can be found in [17].

The state and measurement equations that describe the semiconductor aging model are given below:

$$
\begin{aligned}
& \mathbf{z}_{k}=\mathbf{z}_{k-1} \cdot \exp \left\{-\boldsymbol{\Lambda}_{k}\left(t_{k}-t_{k-1}\right)\right\}+\boldsymbol{\omega}_{k} \\
& \boldsymbol{\Lambda}_{k}=\boldsymbol{\Lambda}_{k-1}+\boldsymbol{v}_{k} \\
& \mathbf{x}_{k}=\left[\mathbf{z}_{k} ; \boldsymbol{\Lambda}_{k}\right] \\
& \mathbf{y}_{k}=\mathbf{z}_{k}+\boldsymbol{v}_{k}
\end{aligned}
$$

where the vector $\mathbf{z}$, consists of the OFF state exponential time decay constants for the IGBT collector-emitter current $\left(\mathrm{I}_{\mathrm{CE}}\right)$, and matrices $\mathbf{C}$ and $\boldsymbol{\Lambda}$ contain their aging decay 
parameters $C$ and $\lambda$ values respectively. The $\mathbf{z}$ and $\Lambda$ vectors are combined to form the state vector $\mathbf{x}$. The measurement vector $\mathbf{y}$ comprises the time decay parameters inferred from measured data. The time index is denoted by $k$. The values of the $\mathbf{C}$ and $\boldsymbol{\Lambda}$ vectors learned from regression can be used to initialize the particle filter. The noise samples $\boldsymbol{\omega}, \boldsymbol{v}$ and $\boldsymbol{v}$ are picked from zero mean Gaussian distributions whose standard deviations are derived from the given training data, thus accommodating for the sources of uncertainty in feature extraction, regression modeling and measurement. System importance resampling of the particles is carried out in each iteration, in order to reduce the degeneracy of particle weights. This helps in maintaining track of the state vector, even under the presence of disruptive effects like unmodeled operational conditions (in our case, high temperatures).

The system description model developed in the offline process is fed into the online process where the particle filtering prognosis framework is triggered by a diagnostic routine. The algorithm incorporates the model parameter as an additional component of the state vector and thus, performs parameter identification in parallel with state estimation. Predicted values of the time decay parameters are compared against end-of-life thresholds to derive time of end-of-life (EOL) and RUL estimates. Figure 1 shows a simplified schematic of the process described above.

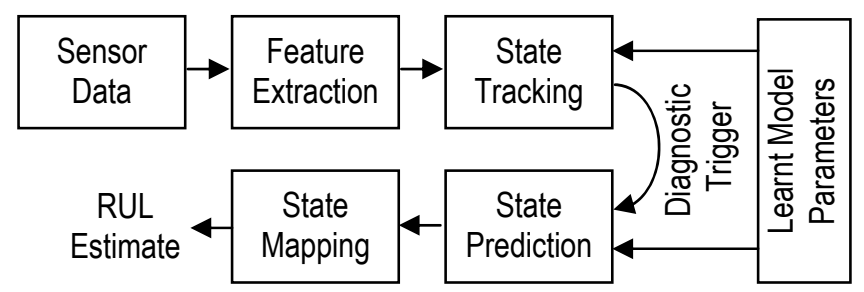

Figure 1 - Particle Filter Framework

\section{EXPERIMENT DETAILS}

The IGBT behaves essentially like a MOSFET and a BJT connected in a Darlington configuration. The input of the IGBT is modeled by the MOSFET, while the output is modeled by a BJT. As a combination of these two devices, the IGBT takes on their characteristics as well as some additional ones caused by their interaction. For example, it exhibits a tail collector-emitter current $\left(\mathrm{I}_{\mathrm{CE}}\right)$ while turning off, which is modeled by a decaying current flowing through the BJT after the MOSFET has been turned off. This is shown in Figure 2.

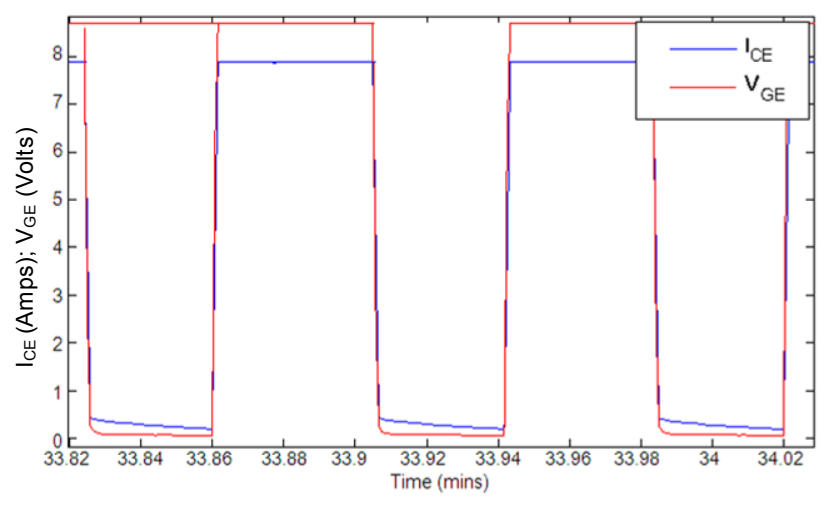

Figure 2 - IGBT Voltage and Current Waveforms

At high temperature thermal cycling these current decay curves change due to hot carrier injection. The trends in these curve shifts can act as a precursor to catastrophic latch-up and subsequent thermal runaway conditions. Figure 3(a)-(c) shows the shift in the OFF-state current decay as aging progresses, finally ending in a latch-up condition as shown in Figure 3(d).
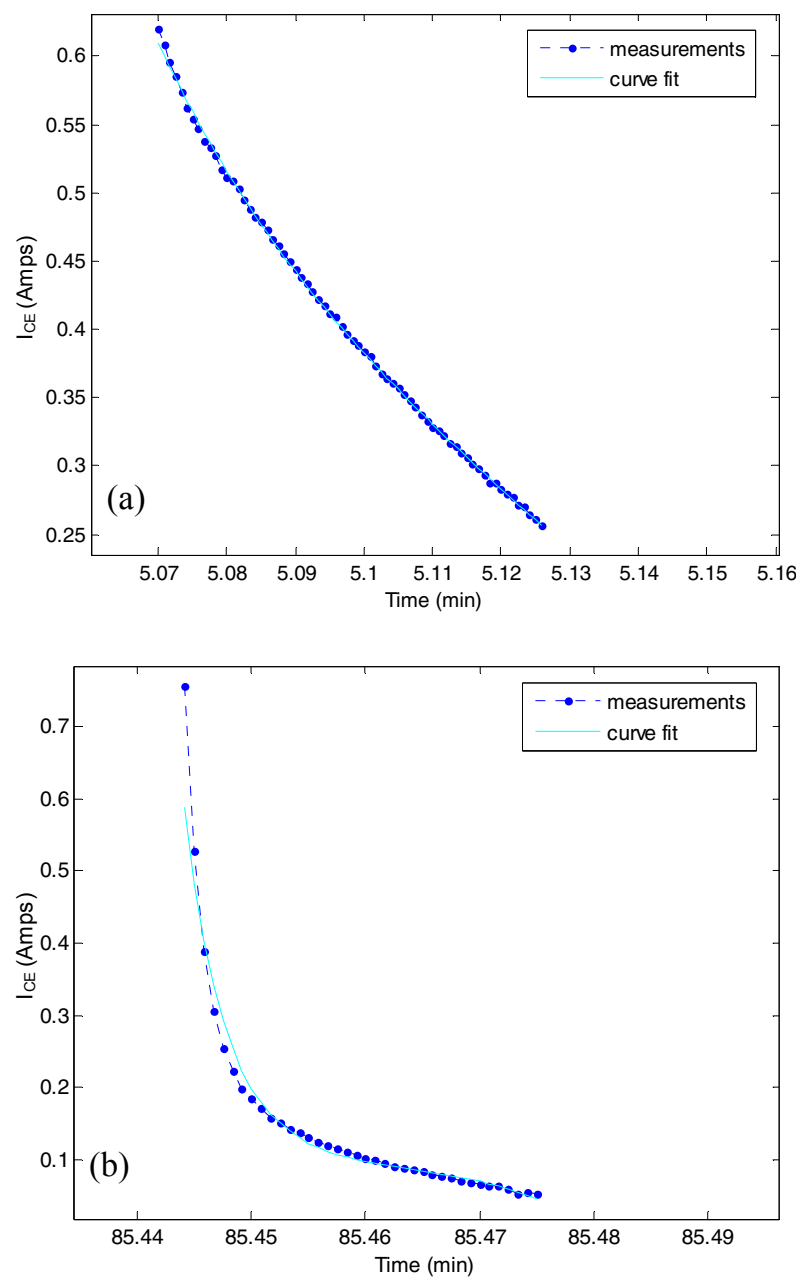

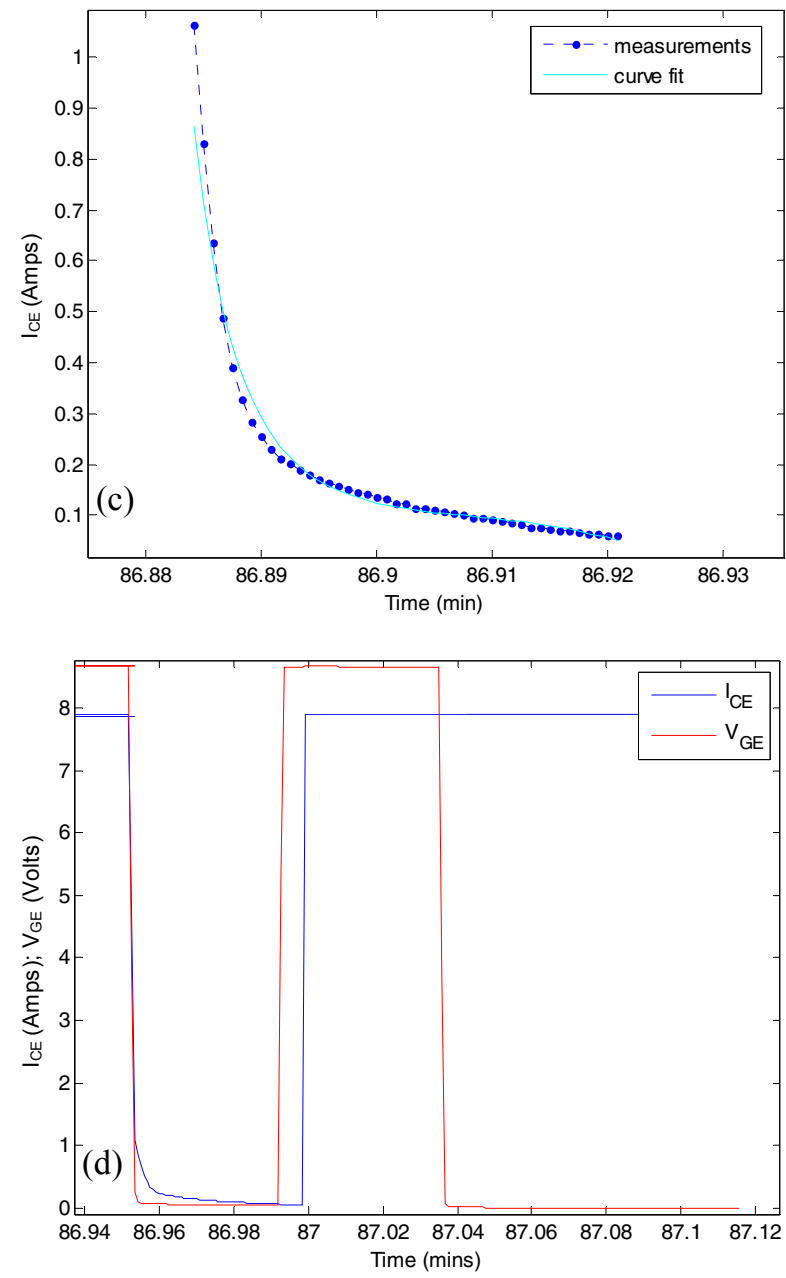

Figure 3 - (a), (b) and (c) Shift in IGBT Off-state Current Decay with Aging; (d) Latch-up Condition

\section{Accelerated Aging Settings}

Thermal overstress accelerated aging was conducted on IGBTs using the agile accelerated aging, characterization and scenario simulation system for gate controlled power transistors presented in [2]. An International Rectifier IRG4BC30KD IGBT with a $600 \mathrm{~V} / 15 \mathrm{~A}$ rating in a TO220 package was aged with no external heatsink. The collectoremitter terminals were connected in series with a load power supply and a $0.2 \Omega$ load resistor. An infrared sensor was used for temperature measurement of the IGBT case. The gate signal was chosen to be $10 \mathrm{~V}$ DC. The aging process was controlled by a hysteresis temperature controller with set points of $268^{\circ} \mathrm{C}$ (low) and $270^{\circ} \mathrm{C}$ (high), switching the gate voltage $\mathrm{ON}$ and $\mathrm{OFF}$ as a control action. The load power supply voltage was $10 \mathrm{~V}$ which resulted in a current of 8A. An additional temperature threshold controller, with a set point of $305^{\circ} \mathrm{C}$, was programmed to turn OFF the load power supply and end the experiment in the event of thermal runaway and latching failures. The IGBT under consideration was aged for $\sim 210$ minutes until latch-up resulting on thermal runaway.

\section{RESULTS}

During the model learning process, we extract the tail current sections from the $\mathrm{I}_{\mathrm{CE}}$ waveform, transform them to the $\log$ domain and then fit $3^{\text {rd }}$ degree polynomials, as expressed below:

$$
\mathrm{I}_{\mathrm{CE}}(t)=\exp \left\{\mathrm{P}_{1} t^{3}+\mathrm{P}_{2} t^{2}+\mathrm{P}_{3} t+\mathrm{P}_{4}\right\} .
$$

The equivalent fits in the time domain are denoted by the cyan lines in Figure 3(a)-(c). The fit parameters $\mathrm{P}_{1}, \mathrm{P}_{2}, \mathrm{P}_{3}$ and $\mathrm{P}_{4}$ are then tracked over the entire aging duration. It is to be noted that the parameters are highly correlated with each other (as denoted by the correlation coefficient matrix shown below in Eqn. 4), and consequently, we use only $\mathrm{P}_{1}$ as the parameter to track.

$$
\rho=\left[\begin{array}{crcr}
1.0000 & -0.9964 & 0.9873 & -0.9747 \\
-0.9964 & 1.0000 & -0.9972 & 0.9899 \\
0.9873 & -0.9972 & 1.0000 & -0.9978 \\
-0.9747 & 0.9899 & -0.9978 & 1.0000
\end{array}\right] \text {. }
$$

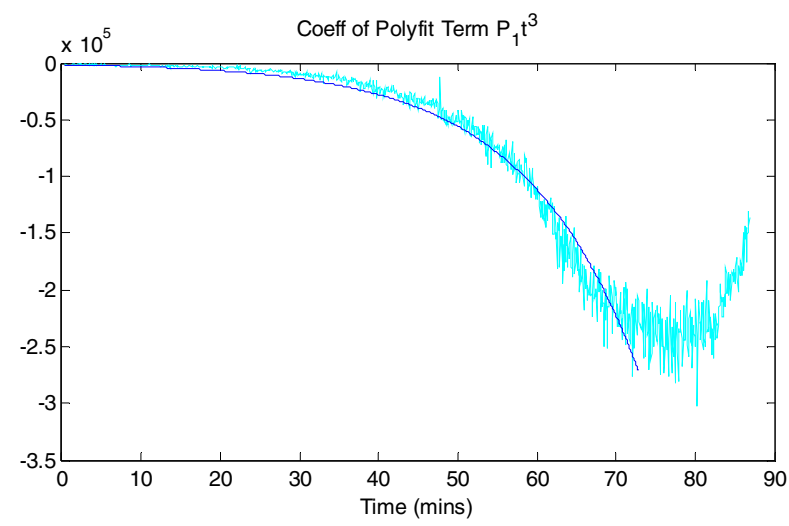

Figure 4 - Trending of Curve Fit Parameter $P_{1}$

Overall, the parameter shows an exponential growth rate (negative) as shown in Figure 4, possibly indicating an Arrhenius aging process. Near the end, the curve shows some anomalous behavior as the IGBT approaches the latch-up condition. Originally the Arrhenius equation described the temperature dependence of the rate of a chemical reaction. Currently it is perceived largely as a empirical relation which can be used to model the temperature variance of many thermally-induced processes.

In running the particle filter framework, we used an exponential growth model for $\mathrm{P}_{1}$ like the one shown in Eqn. 2. Since we did not have separate learning and testing datasets, we did not use the regressed $C$ and $\lambda$ values from the model learning process to jump start our PF algorithm. Instead, we use 0 and 1 as the initial values for $P_{1}$ and $\lambda_{P_{1}}$. The EOL threshold is chosen as $-2.5 \times 10^{5}$, which is approximately the value of $\mathrm{P}_{1}$ at the end of its exponential behavior. Without loss of generality, the prediction time is arbitrarily chosen to be 51.875 minutes. 
Figure 5 shows the performance of the PF in tracking and prediction modes. The inset plot shows the $\lambda_{\mathrm{P}_{1}}$ parameter estimation. After the prediction timeline, the mean of the last $100 \lambda_{\mathrm{P}_{1}}$ values is used for the propagation of each particle according to the state transition model (first line in equation 3). No additional computed $\mathrm{P}_{1}$ values are used to update the particle weights. The propagated particle values are compared against the EOL threshold to compute the distribution of time at EOL. A mixture of Gaussians is then fitted in a least square sense to these EOL values to plot the magenta EOL pdf. The RUL pdf is simply the prediction time subtracted from the EOL pdf. It is to be noted that despite the generic initial particle values, modeling uncertainties and system noise, the PF performance is very good.

\section{Conclusions}

This prognostic exercise, although very preliminary, gives rise to the hope that one can reliably detect failure precursors in semiconductor device behavior and use them in an intelligent prediction framework (e.g. particle filtering) to derive RUL estimates. The fact that the PF provides a RUL pdf, instead of a single MTBF value, makes the interpretation of the prognostic result more useful and intuitive.

There are some significant limiting assumptions employed here: Simple exponential growth models wree used to explain the aging behavior of IGBTs. Although the prediction results are very good, such an approach has limitations when applying the aging parameters and EOL thresholds learnt from one device to another. In order to achieve that, one needs to explicitly derive physics-offailure based aging models, identify their parameters from externally observable device characteristics and then use those models in a PF framework to carry out prognostics. In such a case, aging behavior will be linked to the changes in the internal model parameters which can then be tied to failure mechanisms like hot carrier injection, electromigration etc. The focus of our future work will be to devise aging experiments to fulfill the above objectives.

\section{ACKNOWLEDGEMENTS}

We would like to thank Greg Sonnenfeld for developing the electronics aging testbed. We would also like to acknowledge Patrick Kalgren, Antonio Ginart and Vincent Capra from Impact Technologies LLC, for their work on the NASA Innovative Partnership Program titled "Electronic Prognostics for Critical Avionics Systems".

\section{REFERENCES}

[1] N. Patil, D. Das, K. Goebel, and M. Pecht, "Failure Precursors for Insulated Gate Bipolar Transistors (IGBTs)," in International Conference on Prognostics and Health Management, 2008.

[2] G. Sonnenfeld, K. Goebel, and J. Celaya, "An Agile Accelerated Aging, Characterization and Scenario Simulation System for Gate Controlled Power Transistors," in IEEE AUTOTESTCON, 2008.

[3] A. Ginart, M. Roemer, P. Kalgren, and K. Goebel,

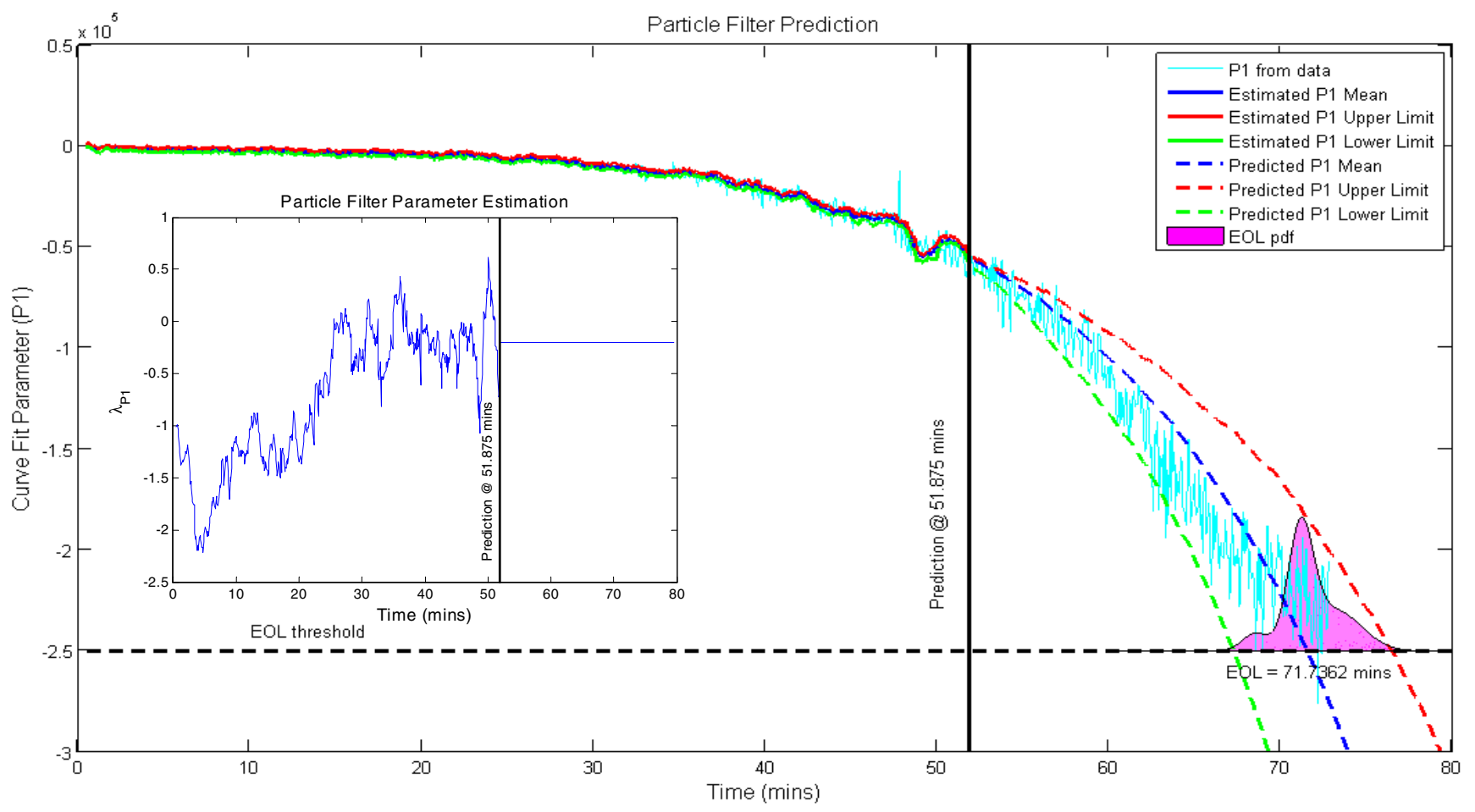

Figure 5 - Particle Filter Tracking and Prediction 
"Modeling Aging Effects of IGBTs in Power Drives by Ringing Characterization," in International Conference on Prognostics and Health Management, 2008.

[4] E. Ameraseka and F. Najm, Failure Mechanisms in Semiconductor Devices, 2nd ed.: John Wiley \& Sons Ltd, 1998.

[5] D. L. Goodman, "Prognostic methodology for deep submicron semiconductor failure modes," IEEE Trans. on Components and Packaging Technologies, vol. 24, March 2001.

[6] W. Wu, M. Held, P. Jacob, P. Scacco, and A. Birolini, "Thermal Stress Related Packaging Failure in Power IGBT Modules," in International Symposium on Power Semiconductor Devices \& ICs, Yokohama, 1995, pp. 330-334.

[7] R. Orsagh, D. Brown, M. Roemer, T. Dabney, and T. Hess, "Prognostic Health Management for Avionics System Power Supplies," in IEEE Aerospace Conference, 2005.

[8] A. Morozumi, K. Yamada, T. Miyasaka, S.Sumi, and Y. Seki, "Reliability of Power Cycling for IGBT Power Semiconductor Modules," IEEE Transactions on Industry Applications, vol. 39, pp. 665-671, 2003.

[9] J. H. Stathis, B. P. Linder, K. L. Pey, F. Palumbo, and C. H. Tung, "Dielectric Breakdown Mechanisms in Gate Oxides," Journal of Applied Physics, vol. 98, 2005.

[10]F. Reynolds, "Thermally Accelerated Aging Of Semiconductor Components," Proceeding of the IEEE, vol. 62, 1974.

[11]C. Diaz, "Automation of Electrical Overstress Characterization for Semiconductor Devices," HewlettPackard Journal, pp. 106-111, October 1994.

[12]M. Trivedi and K. Shenai, "Failure Mechanisms of IGBT's Under Short-Circuit and Clamped Inductive Switching Stress," IEEE Transactions on Power Electronics, vol. 14, 1999.

[13] G. Buh, H. Chung, and Y. Kuk, "Real-Time evolution of trapped charge in a $\mathrm{SiO} 2$ layer: An electrostatic force," Applied Physics Letters, vol. 79, pp. 2010-2012, 2001.

[14] N. Lifshitz and G. Smolinsky, "Hot-Carrier Aging of the MOS Transistor in the Presence of Spin-on Glass as the Inter level Dielectric," IEEE Electron Device Letters, vol. 12, pp. 140-142, March 1991.

[15]A. A. Kuntman, A. Ardal, H. Kuntman, and F. Kacar, "A Weibull Distribution-Based New Approach To Represent Hot Carrier Degradation in Threshold Voltage of MOS Transistors," Solid-State Electronics, vol. 48, pp. 217-223, 2004.

[16] S. Arulampalam, S. Maskell, N. J. Gordon, and T. Clapp, "A Tutorial on Particle Filters for On-line Nonlinear/Non-Gaussian Bayesian Tracking," IEEE Transactions on Signal Processing, vol. 50, pp. 174188, 2002.

[17]B. Saha and K. Goebel, "Uncertainty Management for Diagnostics and Prognostics of Batteries using
Bayesian Techniques," in IEEE Aerospace Conference, 2008.

\section{BIOGRAPHIES}

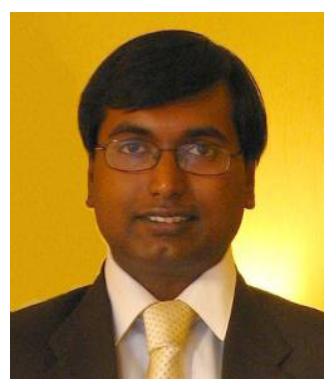

Bhaskar Saha is a Research Programmer with Mission Critical Technologies at the Prognostics Center of Excellence, NASA Ames Research Center. His research is focused on applying various classification, regression and state estimation techniques for predicting remaining useful life of systems and their components. He has also published a fair number of papers on these topics. Bhaskar completed his PhD from the School of Electrical and Computer Engineering at Georgia Institute of Technology in 2008. He received his MS from the same school and his B. Tech. (Bachelor of Technology) degree from the Department of Electrical Engineering, Indian Institute of Technology, Kharagpur.

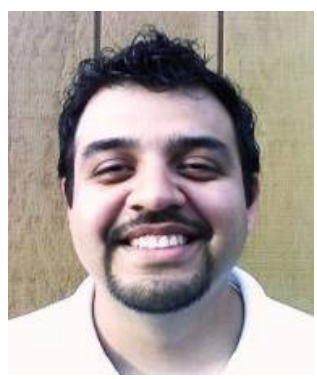

Jose R. Celaya is a visiting scientist with the Research Institute for Advanced Computer Science at the Prognostics Center of Excellence, NASA Ames Research Center. He received a Ph.D. degree in Decision Sciences and Engineering Systems in 2008, a M. E. degree in Operations Research and Statistics in 2008, a M. S. degree in Electrical Engineering in 2003, all from Rensselaer Polytechnic Institute, Troy New York; and a B.S. in Cybernetics Engineering in 2001 from CETYS University, Mexico.

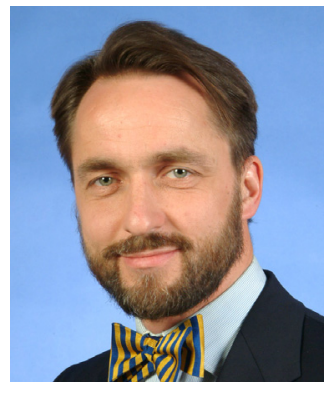

Kai Goebel is a senior scientist at NASA Ames Research Center where he leads the Prognostics Center of Excellence (prognostics.arc.nasa.gov). Prior to that, he worked at General Electric's Global Research Center in Niskayuna, NY from 1997 to 2006 as a senior research scientist. He has carried out applied research in the areas of artificial intelligence, soft computing, and information fusion. His research interest lies in advancing these techniques for real time monitoring, diagnostics, and prognostics. He has fielded numerous applications for aircraft engines, transportation systems, medical systems, and manufacturing systems. He holds half a dozen patents and has published more than 75 papers. He received the degree of Diplom-Ingenieur from the Technische Universität München, Germany in 1990. He 
received the M.S. and Ph.D. from the University of California at Berkeley in 1993 and 1996, respectively.

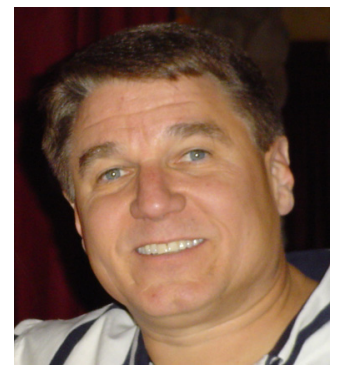

Philip Wysocki is the lead system engineer for the Integrated Vehicle Health Management (IVHM) systems engineering team. He has extensive knowledge and background in test model based data acquisition, as well as programming and system design for diagnostics. He has developed and implemented test design for aging and characterizing IC's and environmental testing. This includes optimization of test hardware and software for prognostics. Phil earned a Bachelor of Science Degree in Computer Science along with over 25 years experience demonstrated at NASA Ames Research Center. 